\title{
Téoros
}

Revue de recherche en tourisme

\section{L'hiver des champs et l'hiver des villes : une manne touristique qui, chaque année, nous fond dans les mains!}

\section{Céline Blais}

Volume 8, numéro 3, novembre 1989

Tourisme hivernal

URI : https://id.erudit.org/iderudit/1080307ar

DOI : https://doi.org/10.7202/1080307ar

Aller au sommaire du numéro

Éditeur(s)

Université du Québec à Montréal

ISSN

0712-8657 (imprimé)

1923-2705 (numérique)

Découvrir la revue

Citer cet article

Blais, C. (1989). L'hiver des champs et l'hiver des villes : une manne touristique qui, chaque année, nous fond dans les mains! Téoros, 8(3), 14-16.

https://doi.org/10.7202/1080307ar 


\section{L'hiver des champs et l'hiver des villes: Une manne touristique qui, chaque année, nous fond dans les mains!}

Le territoire québécois comprend 1667926 $\mathrm{km} 2^{(1)}$ situés au nord du 45 e parallèle. C'est un pays vaste et beau, trahi par les énormes contrastes que les saisons imposent. Bien que les Québécois se plaisent à parler de quatre saisons, il en est une qui se démarque tant par sa rigueur que par sa longueur: l'hiver. C'est une saison qui, du point de vue thermique, est comprise entre la première et la dernière gelée. Sa durée moyenne varie de 120 à 160 jours $^{12 .}$

$\mathrm{Si}$, pour certains, l'hiver est synonyme de plein air, de soleil et de fêttes, d'autres se plaisent ày associer davantage les conditions environnementales du petit écran pour se distraire des longs mois de froidure.

Pourtant, l'hiver n'est plus, grâce à notre modernisme, la dure saison qu'ont connue nos ancêtres. Des maisons mieux isolées et mieux chauffées, un approvisionnement de nourriture plus varié et plus facile d'accès, des véhicules de transport chauffés, etc, ont donné à l'hiver québécois un confort appréciable souvent envié par d'autres pays.

Au moment où la société des loisirs sollicite de plus en plus notre bien-être et notre divertissement quotidien, les progrès consentis à l'hiver nous font découvrir un atout jadis méconnu de nos ancêtres: le tourisme hivernal. D'activités de déplacement obligatoires qu'elles étaient, la raquette, le traineau à chien et la motoneige se sont élevés au rang d'activités de loisirs.

Cet article souhaite présenter quelques éléments de réflexion afin de questionner la place de l'hiver dans les infrastructures touristiques québécoises. De retour des pays Scandinaves, l'auteure se permet quelques comparaisons.

\footnotetext{
- Madame Celine Blais est professeure de géographie su CEGEP de Sherbrooke. Bacheliere en Gestion et intervention touristiques et maitre en géographie, elle est l'auteure et l'organisatrice len collaboration avec le C.A.T.I.) du colloque sur le Tourisme hivernal qui s'est. tenu à I"U.Q.A.M. en février 89 .
}

\section{L'impitoyable hiver}

L'enrichissement des personnes et l'amélioration des conditions de vie hivernales ont eu un curieux effet sur les Québécois et les Québécoises! Au lieu de mieux apprécier cette douce conquête de dame nature, les gens semblent s'être rebel$l e ́ s$, maudissant le froid et la neige comme un fléau. Avec leurs habits prêtt-ă-porter, les malheureux ont vite sauté dans leur "prêt-à-partir" vers les contrées plus chaudes. Là au moins, pas de gadoue et pas de sous-zéro! Mais cecomportement n' indique que la pointe de l'iceberg. Car, plus près de la vie quotidienne urbaine, ce sont des centaines de comportements ou d'infrastructures qui languissent faute de reconnaissance hivernale. Et pourtant, peuton croire que seul le territoire québécois est pris d'assaut par un refroidissement saisonnier? On estime à près de deux milliards la population vivant dans cette partie de la terre où l'isotherme de janvier correspond à $5^{\circ} \mathrm{C}$, et à un milliard, la population correspondant à l'isotherme de $0^{a} \mathrm{C}$ en janvier. Cela signifie pas moirs de 650 villes d'une population de 100000 habitants et plus ${ }^{(3)}$. Voilà un large potentiel d'échanges ou d'idées concernant l'architecture, les activités, les politiques, les infrastructures propres à l'hiver. S'il est vrai qu'il fait si froid et qu'il neige tant (en douterait-on?), pourquoi n'exploite-t-on pas à sa juste mesure cette réalité naturelle qu'est l'hiver?

Il fut un temps où l'hiver au Québec êtait synonyme de saison des fêtes, de divertissements. C'était la saison des menus travaux mais aussi des longues veillées d'histoires. La maison devenait alors le centre du monde. C'était l'époque où le Québec ne connaissait pas les 14 soleils. Les temps ont bien changé, mais le temps qu'il fait sur le pays, lui, ne bouge pas. A l'aube d'un réchauffement de la planète, l'hiver demeure solidaire de la terre québécoise. Si nos ancêtres avaient réussi à apprivoiser cette rude saison, les citadins que nous sommes devenus, la boudent beau temps, mauvais temps. Pourtant, $c^{\prime}$ est au Québec que l'hiver est le plus ensoleillé(4), en Occident etc'est ici qu'y vit la population nordique la plus latine.

Comment expliquer ce refus collectif d'accepter et d'intégrer l'hiver dans toutes les infrastructures et activités quotidiennes de décembre à mars? Comment le Québec et en particulier Montréal peuvent-ils ignorer cette manne touristique qui pourrait chaque année vivifier les intrants touristiques pendant la "morte" saison. La problématique entourant cette question est, selon l'auteure, complexe et variée.

\section{L'hiver en perdition: I'enjeu du progrès}

Nos ancêtres avaient remarqué que les champs recouverts d'un épais manteau de neige, produisaient plus de foin l'été suivant, que ceux balayés par le vent durant tout l'hiver. $^{(5)}$

Ces derniers attribuaient même des propriétés fertilisantes à la neige. On peut dire que la neige, sans fertiliser, protège la terre du gel et lui permet de regagner plus rapidement sa fertilité première ${ }^{(5)}$. Mais l'hiver des champs nous confronte de moins en moins. C'est en ville que vit la très grande majorité de la population québécoise et c'est dans cet univers urbain que chacun de nous négocie quotidiennement son bien-être.

Une si froide saison oblige à des préparatifs. De la tenue vestimentaire à la maison, en passant par les moyens de transport, l'hiver est une saison exigeante. Loin d'être une barrière au développementéconomique, l'hiver constitue une stimulation, car de nombreux coûts sont directement associés à l'hiver.

Le niveau de vie des Québécois s'est considérablement amélioré depuis ces trente dernières années. Mais ce n'est pas sans bousculer notre rapport avec $l^{\dagger}$ environnement ou le rythme des saisons que nous bénéficions aujourd'hui de cette aisance matérielle. Ainsi le frigo a remplacé le caveau et l'abondance de fruits et légumes à longueur d'année nous a fait oublier qu'il 
$y$ avait une saison verte et une saison blanche.

Nos habitudes de consommation urbaine nous ont fait croire à la facilité: des fraises à l'année, un chauffage central, des jardins intérieurs, l'été à longueur d'année quoi! L'hiver n'est pas une saison incitant au geste spontane. On ne peut se permettre de vivre au jour le jour. Le froid nous apprend à prévoir, à se débrouiller, à inventer. Il réclame une ingéniosité constante pour la maison, la nourriture, le vêttement, le transport et pour le tourisme.

\section{Et I'environnement?}

L'environnement nordique est fragile. Aussi, la vastitude du Québec est-elle mal servie? Loin de commander l'anénagement rationnel du milieu, c'est tout le territoire qui est pris en otage par une absence de respect et de planification adéquate.

Si on s'est plu à couper les arbres de notre jardin parce qu'ils cachaient la vue, quel sort a-t-on réservé à ce million de lacs et de rivières laissés par une ère glaciaire généreuse? Les cours d'eau du Québec sont pollués à différents degrés et sont, en plus, inaccessibles. La nature est, au Québec, privée: à chacun son bout de lac, de terre et "cadastre oblige sa clôture." II n'est pas étonnant de constater que ce comportement individualiste avantage la pollution et la détérioration de l'environnement tout entier. Les Suédois jouissent à cet effet d'une loi et d'une éducation qui favorise la prise en charge collective de l'environnement. Le droit d'accès commun qui relève du droit coutumier permet à chacun de se déplacer librement dans la nature à condition de ne pas endommager les biens d'autrui. De plus, une aire de 300 mètres autour des cours d'eau est strictement publique. Chacun, chacune peut se baigner ou canoter où bon y chante.

Quoi de plus naturel que de profiter de ce qui est abondant chez-soi! Malheureusement, c'estce qui choque le plus au Québec: des hectares de forêt et de champs impénétrables parce que clôturés, des cours d'eau barricadés de chalets privés et un fleuve qui, à Montréal, n'est accessible que sur une carte. Voilà la lourde réalité quotidienne à laquelle est confrontée non seulement la population du Québec mais aussi tous ceux et toutes celles qui revvaient de visiter cette terre d'abondance.

L'environnement québécois, aujourd'hui peu accessible, rend $\mathrm{d}^{\dagger}$ autant plus difficile la vie hivernale, qu'il confine la population chacun chez soi au lieu de lui favoriser un rapport convivial avec ses voisins. Pourtant, c'est la grande sociabilité des Québécois et leclimat froid qui a fait naître, il y aquelques décennies, un type de peuplement rural facilitant le voisinage.

L'hiver qui fait rềver surd'autres continents les adeptes d'espaces blancs est-il en train de devenir une saison morte, otage de notre développement malsain?

\section{Le mal de l'hiver}

Si le Québec a mal à son tourisme hivernal, on peut alors s'interroger car pourrait-on douter ici de l'abondance de la matière première?

Quels aménagements ou infrastructures choisissons-nous pour bien mettreen valeur le Québec auquel la population québécoise s'identifie? L'architecture, les événements culturels, les politiques québécoises \$'harmonisent-ils avec notre réalité franconordique?

Les infrastructures avantageuses pour les contrées du sud favorisent-elles nécessairement celles du nord? Par exemple on peut questionner la très lourde présence du réseau routier dans les villes d'hiver. Là où des sentiers pédestres ou des pistes de ski auraient bonifiés et humanises $1^{\dagger}$ espace urbain, on retrouve cette présence démesurée de l'automobile.

Et puis, tout comme si l'automobile avait tracé la route à d'autres importations américaines ou sudistes, on a accepté dans notre environnement des projets, valables peut-être pour la courte saison d'été mais qui ne favorisaient guère la saison hivernale. Dans quelles mesures a-t-on calqué le développement $\mathrm{d}^{*}$ attraits touristiques qui ne font qu ${ }^{\dagger}$ accentuer la paralysie hivernale? Les glissades d'eau, les parcs d'attractions et autres ne présentent pas de caractère polyvalent pendant la saison froide. Ils atrophient les paysages avec leurs tubulures. métalliques géantes.

Aussi l'hiverquébécois souffre-t-ild'abord d'infrastructures inadéquates (culturelles et physiques) pour animer cette longue et fascinante saison. Paradoxalement, c'est en hiver que l'individu a le plus besoin d'une organisation facilitante d'activités de voisinage et de plein air. Les qualités intrinsèques des autres saisons sont de nature à multiplier les contacts avec des activités extérieures et la population.

L'hiver québécois réclame donc des

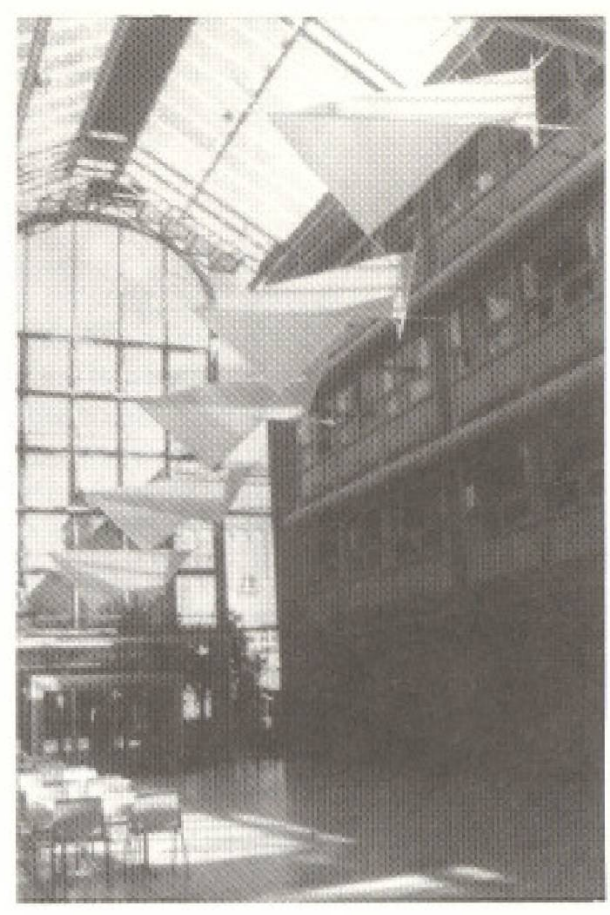

infrastructures et des aménagements à la mesure de ses qualités géographiques, démographiques, culturelles et linguistiques.

\section{Intégrer sa nordicité}

C'esten utilisant les forces de l'hivercomme alliées que l'on découvre le potentiel de cette saison. Je cite en exemple l'université de Trondheim en Norvège, où différents bâtiments ont été réunis par des murs et des toits vitrés. L'intégration du verre permet de manger, d'étudier et de circuler sous le soleil d'hiver.

Au Québec, des efforts louables en ce sens gagnent du terrain. Malheureusement, de telles architectures sont encore trop associées à des environnements de luxe tels le complexe résidentiel "Tropique Nord" ou les Courts Mont-Royal à Montréal.

L'attrait touristique veut amener le visiteur à connaître de nouveaux espaces mais aussi à générer des deniers dans les fonds du pays visité. Or, est-il important de le préciser tout attrait touristique, pour être valable, doit initialement appartenir aux modes de vie ou à des éléments significatifs auxquels s'identifie l'hôte. C'est ainsi que la Vasaloppet, cette course internationale de ski de fond qui réunit chaque année plus de 10000 participants venus de par le monde s'identifie d'abord à la Suède. Cette épreuve de ski commémore le retour de Gustave Vasa qui emprunta ce parcours dans les forêts de Dalécarlie après avoir fui des Dannois en 1521. Danslatradition suédoise, 
on boit le Blabers Soppa (breuvage aux bleuets) et les futurs gagnants de la course ont droit à la "kranskulla", cette couronne de fleurs déposée sur la tête, 400 mètres avant l'arrivée.

Dans la région de Trondheim, c'est avant tout par souci de commodité que l'on a éclairé les pistes de ski de fond avoisinantes. Un peu plus de lumière permet aux Norvégien-ne-s de se balader plus tardivement mais aussi d'aller et de revenir du travail en ski. $\mathrm{A}$ une latitude où les heures de soleil se font restreintes en hiver, une telle initiative bonifie la qualité de vie quotidienne des résidents tout en stimulant la venue des touristes.

Au Québec, malheureusement, des projets d'hiver intégrés au patrimoine et à la nature québécoise tardent à venir. Non pas que les efforts de développement ne sont pas souhaités mais certains, tel le développement de pistes de motoneige comme source d'attrait touristique, commandent la réflexion. Comment expliquer que les autorités québécoises tententde promouvoir de tels projets alor's que la Norvège et la Suède interdisent l'utilisation de la motoneige (sauf en cas d'urgence).

Serait-il vrai de croire que certains pays intègrent mieux leur nordicité que d'autres? Pourquoi le Québec se toume-t-il plus souvent vers des projets de prolongation de l'été (Beach party, vagues et dôme pour les lacs, etc.) plutôt que vers des projets d'intégration de l'hiver?

Les Finlandais qui n'ont pas invente le sauna l'ont cependant développé à un point tel qu'il est devenu déterminant pour leur mode de vie: une chaleur bienfaisante pendant les jours froids et sombres. L'authentiquesauna finlandais est construit et chauffe au bois. Il est situé près d'un lac, de la mer ou d'une rivière où on peut nager après avoir bien transpiré été comme hiver.

Le sauna est synonyme de purification, de détente, de délassement et de guérison. Il est de plus considéré comme une introduction et une préparation aux dimanches et aux jours de fête. L'hiver peut donc être une saison chaleureuse.

Même au Québec la saison froide n'a jamais été une saison de réclusion. Parce que les travaux des champs libéraient nos ancêtres, l'hiver était la saison où "on avait le temps" pour se retrouver, se visiter, se courtiser. $C^{\prime c}$ tait le temps des grandes bouffes cuisinées. Les infrastructures du temps; la maison, la famille, l'église, prenaient alors

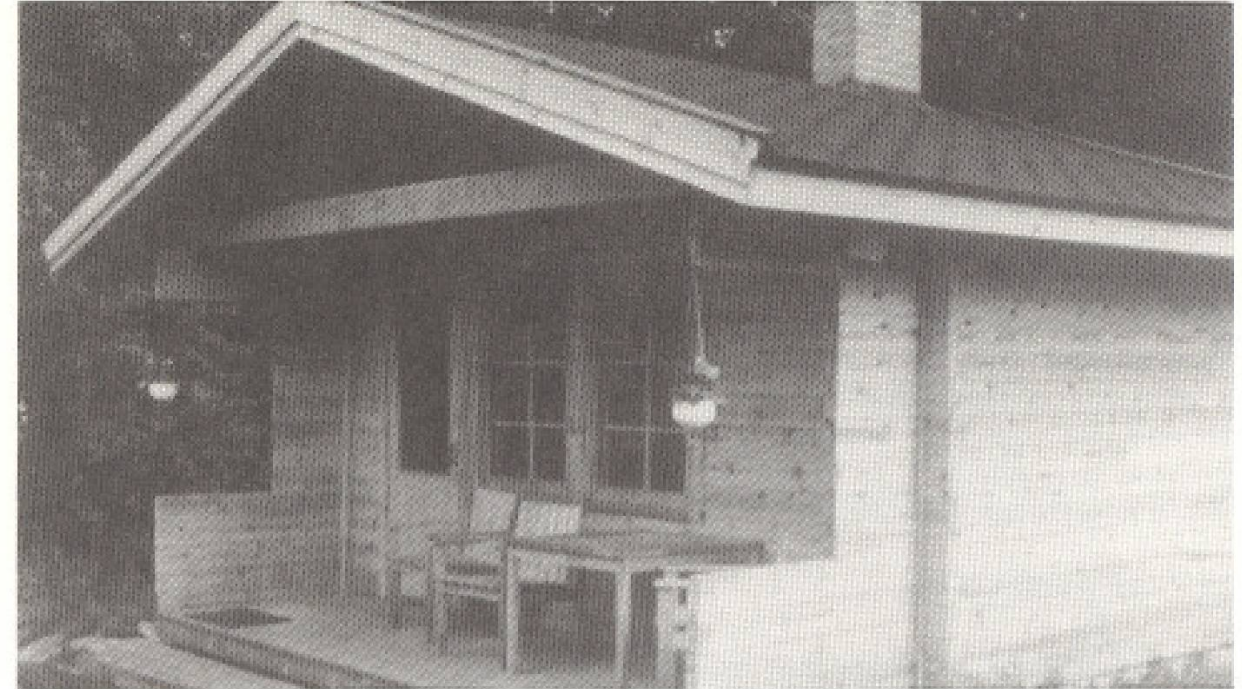

toute leur importance.

Pour la grande ville d'aujourd 'hui, l'hiver est aussi la saison rêvée pour y decouvrir "une autre ville" sous la neige, mais aussi de nouvelles activités culturelles. Des expositions ou des festivalsqui privilégient les salles intérieures se font trop rares dans la froide saison.

\section{Des vacances chez-soi l'hiver: pourquoi pas?}

En conclusion, il faut garder ă l'esprit que plusieurs québécois et québécoises cherchent des altematives valables à une fuite répétée vers le soleil.

Par ailleurs, si plusieurs arrivent ả profiter par le ski ou la raquettedu calme bienfaisant de la neige, plusieurs ne partent pas et restent près de leurs lieux de travail, sept jours sur sept.

Il apparaît de plus en plus urgent que les. autorités municipales se préoccupent des gens qui ne partent pas chaque finde semaine ou qui tout simplement ne partent pas en vacances l'hiver. Partout dans les villes, il existe des centaines de possibilités de passer quelques jours, voire plus, à la découverte de son milieu. Notre hiver est en ville et il devient urgent que l'on développe une politique du loisir hivernal adapté au quotidien des citoyens.

Si les promoteurs touristiques, les autorités gouvemementales et la population même s'accordent pour donner à l'hiver une place de choix dans tous les domaines concemant la vie quotidienne, peut-être vivrons-nous enfin toutes les splendeurs et la force de la nordicité québécoise.

Est-il permis de réver que les francofolies de décembre 90 provoqueront sur Montréal non seulement une "poudrerie" de rock francophone, mais aussi laisseront place à d'autres présentations artistiques, cinématographiques et musicales avec neige sous le vent. La juxtaposition des grands évếnements culturels et artistiques avec la saison froide permettrait peut-être à Montréal de se gagner de nouvelles clienteles.?

\section{Notes explicatives}

(1) Excluant le Labrador depuis le trace de 1927

(2) PROVENCHER, Jean, C'était I'hiver, la vie rurale traditionnelle dans la vallèe du St-Laurent.

(3) The Economist Publieations Limited, The Business of Winter, Winter Cities Showcase 1988. p. 1.

(4) Sclon Environnement Canada, on compte une movenne de 80.4 heures (station McGill) densoleilement pour lo mois do decembre a Montrobal, alors que la movenne pour Paris est de 40 heures, pour Stockholm 26 heures et pour Helsinki, 17 heures.

(5) PROVENCHER, Jean, C'etait I'hiver, la vie rurale traditionnelle dans la vallée du St-Laurent. Boréal, 1986, p. 22.

\section{Refórences}

ALEXANDRE. Normand et al Les défis de Pamenagement, textes revues et edites par Denis. Vaugois, Assemblee Nationale du Quebec, 1982.

DEFFONTAINES, Pierre, L'homme et l'hiver au Canada, Gallimard, 1957.

GÁLAND, Bertil, Le nord en hiver, parcours du haut de I"Europe de Reykjavik à Moscou, edition 24 heures. Lausanne, 1985.

KRIPPENDOFF, Jost, Les vacances et après? Pour voyages, I'Harmahan, 1987.

LAMONTAGNE, Sophie-Laurence, L'hiver dans la

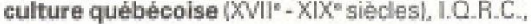
1983.

LYNCH, Kevin, Voir et planifier' l'amenagement qualitatif de I'espace, Bordas, Paris 1982.

PRONENCHER, Jean, C'était I'hiver, la vie nurale traditionnelle dans la vallé du St-Laurent, 1986.

RIOUX, Mercel, Les Québécols, Seuil, 1974.

The Economist Publications Limited. The Business of Winter, Winfer Cites Showcase, 1988. Bcreal, 1986, p. 12 . une nouvelle comprehension des loisirs et des 